\title{
FERRAMENTA DIGITAL PARA DIVULGAÇÃO CIENTÍFICA EM ENGENHARIA DE MATERIAIS: CANAL DO YOUTUBE ENGENHEIROS DO AMANHÃ
}

Leonardo Almeida Freire - leo.a.freire@hotmail.com Universidade Federal de Lavras, Departamento de Engenharia 37200-900 - Lavras - Minas Gerais

Dafny Peres Candido Souza-dafny.souza@gmail.com Universidade Federal de Lavras, Departamento de Engenharia 37200-900 - Lavras - Minas Gerais

Mirna Maria Machado Diniz-mmmd@estudante.ufla.br Universidade Federal de Lavras, Departamento de Engenharia 37200-900 - Lavras - Minas Gerais

Júlio César Ugucioni-julio.ugucioni@ufla.br Universidade Federal de Lavras, Departamento de Física 37200-900 - Lavras - Minas Gerais

Juliano Elvis Oliveira - juliano.oliveira@ufla.br Universidade Federal de Lavras, Departamento de Engenharia 37200-900 - Lavras - Minas Gerais

Resumo: O presente trabalho tem como objetivo apresentar os resultados (alcance e perfil do público) obtidos através da produção de conteúdo de divulgação científica do curso de engenharia de materiais. Todo conteúdo produzido foi publicado na plataforma digital "youtube". Este trabalho mostra o alcance e perfil do público que acessa o canal do youtube "Engenheiros do Amanhã". Ferramentas digitais se mostraram eficientes para divulgar conteúdos de engenharia para público jovem e estudantes recém ingressos no curso de engenharia de materiais de forma a reduzir a evasão nos cursos de engenharia e maior conscientização da população sobre o papel do engenheiro na sociedade brasileira. Este trabalho foi desenvolvido em torno da produção e publicação de conteúdo áudio visual para o canal do youtube "Engenheiros do Amanhã", sendo que todo o conteúdo como produzido por estudantes recém ingressos do curso de Engenharia de Materiais da Universidade Federal de Lavras, no período entre 2017 e 2019. A experiência relatada se baseia na descrição do processo de produção dos materiais e dos trabalhos realizados nos laboratórios, em casa e durante algumas aulas expositivas ministradas pelo professor responsável da disciplina. Este trabalho apresenta dados importantes referentes as visualizações, que podem ser utilizados para desenvolver e aplicar estratégias de difusão dos conteúdos da engenharia de maneira lúdica e eficiente.

Palavras-chave: Divulgação científica. Plataformas digitais. Difusão do conhecimento. Engenharia de materiais 


\section{INTRODUÇÃO}

Atualmente, o fenômeno da evasão no ensino superior, caracterizado pela interrupção no ciclo de estudo (GAIOSO, 2005), vem preocupando as instituições de ensino. Essa alta evasão também atinge a cursos de engenharia e ocorre normalmente semestres iniciais de cada curso.

Estudos realizados pela Comissão Especial para o Estudo da Evasão (BAGGI, LOPES, 2010; PEREIRA, et al., 2006; SILVA, CECÍLIO, 2007) indicaram que as principais causas de evasão de alunos estão ligadas ao mercado de trabalho, ao reconhecimento social da carreira, a qualidade do ensino fundamental e médio, a razões econômicas e políticas, e finalmente a compreensão prévia da carreira, sendo a solução dessa causa o principal objetivo desse trabalho.

A universidade tem como papel, além de realizar pesquisas e atuar formação de profissionais, realizar projetos de integração com a sociedade para que o conhecimento adquirido na universidade não se restrinja aos integrantes da instituição, e para que se desperte um interesse pela ciência e engenharia na população em geral (FERNANDES, 2009). Além disso, a divulgação científica também pode atuar dentro das instituições de ensino, incentivando os autores a produzirem mais artigos em diferentes temáticas, sendo esse aumento na produtividade científica média por autor, um grande desafio a ser resolvido (LIMA, VIANA, 2017).

Existem várias ações que possibilitam a divulgação de conteúdo dos cursos de engenharia, como palestras em escolas, eventos abertos ao público e difusão de informação por diversos meios de comunicação. Nesse projeto, teve-se como objetivo a divulgação do curso de engenharia de materiais, curso relativamente novo e ainda pouco conhecido no sul de Minas Gerais.

Como estratégia de divulgação, foram adotadas palestras em escolas, produção de folders e cartazes, produção de vídeos para o Youtube e apresentação no evento "UFLA de portas abertas", onde foram recebidas mais de 17 mil pessoas de 220 escolas, segundo a Universidade Federal de Lavras (UFLA).

A plataforma a digital escolhida para hospedar o conteúdo áudio visual foi o Youtube devido a sua enorme popularidade e importância para as novas gerações. Sendo a mais famosa do mundo com foco em compartilhamento de vídeos (QUINTANILHA, 2017), a plataforma foi fundada em fevereiro de 2005 e segundo a Omnicore, em 2020 conta com números exorbitantes, como 2 bilhões de usuários mensalmente ativos e 30 milhões de usuário diariamente ativos (ASLAM, 2019).

Dentre os criadores de conteúdo para o Youtube, vários optam por produzir conteúdo voltado pra divulgação cientifica e tem alto retorno no que diz respeito à retenção de público, como o canal "Nerdologia", com 2.81 milhões de pessoas inscritas para receberem atualizações (IAMARINO, 2010) e o "Canal do Pirula", com 879 mil inscritos (NASCIMENTO, 2006). 


\section{METODOLOGIA}

O projeto foi baseado num modelo de divulgação científica que tem como objetivo decodificar termos e jargões técnicos de temas de engenharia e associá-los a temas do dia a dia do público alvo (jovens e adolescentes). Essa decodificação foi feita por estudantes do curso de engenharia de materiais da Universidade Federal de Lavras e teve por objetivo democratizar o conhecimento científico e tecnológico, além de motivar o aluno de engenharia na busca de conhecimentos. A partir disso, o desenvolvimento projeto de implementação foi organizado em quatro etapas iniciais: a) identificação dos conteúdos relevantes, b) elaboração de um roteiro de gravação, c) revisão do conteúdo por professores da área e d) gravação do material e disponibilização no canal do Youtube "Engenheiros do Amanhã".

Como exemplo do material produzido, segue abaixo o roteiro produzido para o primeiro vídeo "O que é a engenharia de materiais?".

Bom dia, meu nome é Leonardo Freire e vim apresentar pra você que está pensando em qual carreira seguir e pra você que é simplesmente curioso meu curso, a engenharia de materiais.

A engenharia de materiais é a engenharia que conecta a ciência e a engenharia para desenvolver novos materiais pra indústria.

O foco principal dessa engenharia é tanto criar um novo material com propriedades novas ou otimizadas que supram a necessidade de algum ramo de desenvolvimento tecnológico ou a produção dos materiais já empregados no setor produtivo de forma a atender todas as demandas da sociedade.

Para ilustrar nosso objetivo, tomarei como exemplo as projeções do Elon Musk, empreendedor dono da empresa SpaceX.

O Elon Musk, que eu considero o Homem de Ferro da vida real, além de diversos planos e desenvolvimentos de alta tecnologia, pretende colonizar marte.

Acreditando na proposta dele, pensemos o que é necessário para tal feito.

A princípio, para um foguete sair da orbita da terra e chegar em outro planeta é preciso que ele carregue combustivel suficiente para essa trajetória. O problema é que quanto mais combustível é levado, mais pesado o foguete fica, e mais combustível é necessário. Atualmente, esse problema é solucionado eliminando partes do foguete conforme os tanques se esvaziam. Mas e se além de ir para outro planeta, você quiser voltar para a terra? Os tanques já teriam sido eliminados, então essa mesma técnica pode não ser o ideal.

Um modo de viabilizar essa viagem seria desenvolver um material mais leve para o foguete, de forma que sua resistência à gravidade zero e temperaturas muito baixas do espaço se mantenha intacta.

É aí que entra a engenharia de materiais. Trabalhamos todos os dias tentando otimizar os materiais existentes e criar novos materiais para expandir o nosso universo tecnológico.

Mas a engenharia de materiais não está presente somente em tecnologias de ponta. Podemos atuar em qualquer área que podemos imaginar, pois tudo envolve um material.

Para construções civis é necessário bons materiais que suportem toda a estrutura da construção, além de materiais que evitem infiltrações e por que não amenizem a temperatura no interior da casa?

Para componentes eletrônicos é essencial que a informação seja transmitida sem perdas de dados e sem que o componente se degrade com um aumento de temperatura ou desgaste da peça. A fibra óptica é um ótimo exemplo de um desenvolvimento de um material que 
evite a perda de dados, e dentre outras coisas, esse é o motivo dela ser tão mais rápida e efetiva.

Também podemos atuar na área médica.

Próteses, por exemplo, precisam ser feitas de um material resistente ao impacto, corrosão e ao mesmo tempo que seja biocompativel.

Uma preocupação frequente na engenharia de materiais é reduzir o impacto que a tecnologia causa ao meio ambiente.

Podemos tomar como exemplo o plástico, que hoje é um dos maiores problemas em termos de poluição. Porém existem novos materiais plásticos que são biodegradáveis. Ou seja, caso seu descarte seja feito da forma correta, microrganismos poderão metabolizar esse material. E assim reduzir o impacto ambiental em aterros sanitários e lixões.

E é por isso tudo e diversas outras coisas que eu convido você que está me ouvindo a se juntar a nós e contribuir para o desenvolvimento de novas tecnologias que realmente possam mudar o mundo.

Na descrição do vídeo eu vou deixar um link para a página da Engenharia de Materiais da UFLA - Universidade Federal de Lavras que está incentivando esse projeto e fazendo com que você passe a ter esse conhecimento.

Até mais e espero te ver num futuro próximo.

O projeto envolveu as seguintes etapas: $1^{\circ}$ identificação de temas de interesse ao público jovem; $2^{\circ}$ elaboração de roteiros contendo conceitos de ciência dos materiais e temas da cultura pop; $3^{\circ}$ gravação e edição do conteúdo; $4^{\circ}$ disponibilização do conteúdo produzido na plataforma youtube e acompanhamento do alcance e engajamento dos vídeos. Além disso, há uma concorrência pela atenção do usuário devido à quantidade de informações de fácil acesso pela internet (VELHO, 2011). Dessa forma, é importante que o conteúdo apresentado seja bem planejado, suprindo a necessidade de informações rápidas, e fornecendo entretenimento para os usuários da plataforma. Após a criação e revisão detalhada de cada roteiro, foram iniciados os processos de gravação do vídeo e áudio, onde um locutor, com a ajuda de um gravador áudio e vídeo, ficou encarregado de fazer a narração dos roteiros e a gravação dos vídeos.

\section{RESULTADOS E DISCUSSÃO}

O primeiro vídeo produzido foi o "O que é a engenharia de materiais?" (https://www.youtube.com/watch?v=QRTjxJdr2Fw\&t), explicando o que faz um engenheiro de materiais e qual a importância do curso de maneira prática. O vídeo, até o dia 10/04/2020, apresentava 10459 visualizações.

Em sequência, foi produzido o vídeo "Materiais cerâmicos - Engenharia de materiais" (https://www.youtube.com/watch?v=-3EDT9JL5AU\&t), na tentativa de tentar introduzir conceitos importantes do curso de maneira básica. Tendo esse vídeo 7208 visualizações até o dia 10/04/2020.

Em uma tentativa de abordagem diferente, foi produzido o terceiro vídeo, de título "Como vencer uma guerra infinita com engenharia" (https://www.youtube.com/watch?v=PzsoXVTVys), surgido da ideia de aproveitar a popularidade do filme "Vingadores, guerra infinita", teve como objetivo a produção de um conteúdo de entretenimento com ciência, onde os ouvintes estariam aprendendo conceitos da engenharia de materiais enquanto assistiam a um tema lúdico, que estava em alta no momento da postagem. 
Esse vídeo apresentou 1030 visualizações até o dia 10/04/2020. Porém, esse vídeo em especial foi compartilhado no Facebook através da página da Universidade Federal de Lavras, onde atingiu 2500 visualizações até o dia 10/04/2020.

A partir da ferramenta analytics do Youtube, é possível fazer algumas análises importantes. A primeira análise realizada se trata da quantidade de acessos por dia para cada um dos vídeos. As curvas foram traçadas utilizando dias corridos para que a observação dos eventos fosse facilitada.

O primeiro vídeo a ser analisado foi o "O que é a engenharia de materiais". Sendo a curva referente a esses dados apresentada na figura 1. Para esse vídeo, as análises começaram a partir do dia 14/01/2018 (data de postagem do vídeo), e terminaram no dia 08/04/2020.

A partir dos dados, observa-se um comportamento de pico de acessos nos meses de janeiro, podendo ser justificado pela busca sobre o tema durante a decisão de qual curso se matricular, realizada pelos alunos. Outros picos menores podem ser vistos no mês de junho, referente ao período de escolha para os alunos que não entraram no início do ano e tentaram novamente no segundo semestre letivo do ano. A menor quantidade de visualizações no meio do ano em relação ao início pode ser justificada pela possibilidade de os alunos já terem pesquisado sobre os cursos no início do ano, quando tentaram pera primeira vez, e já terem definido sua escolha.

Figura 1: Efeito do tempo no número de visualizações dos vídeos postados no canal "Engenheiros do Amanhã"

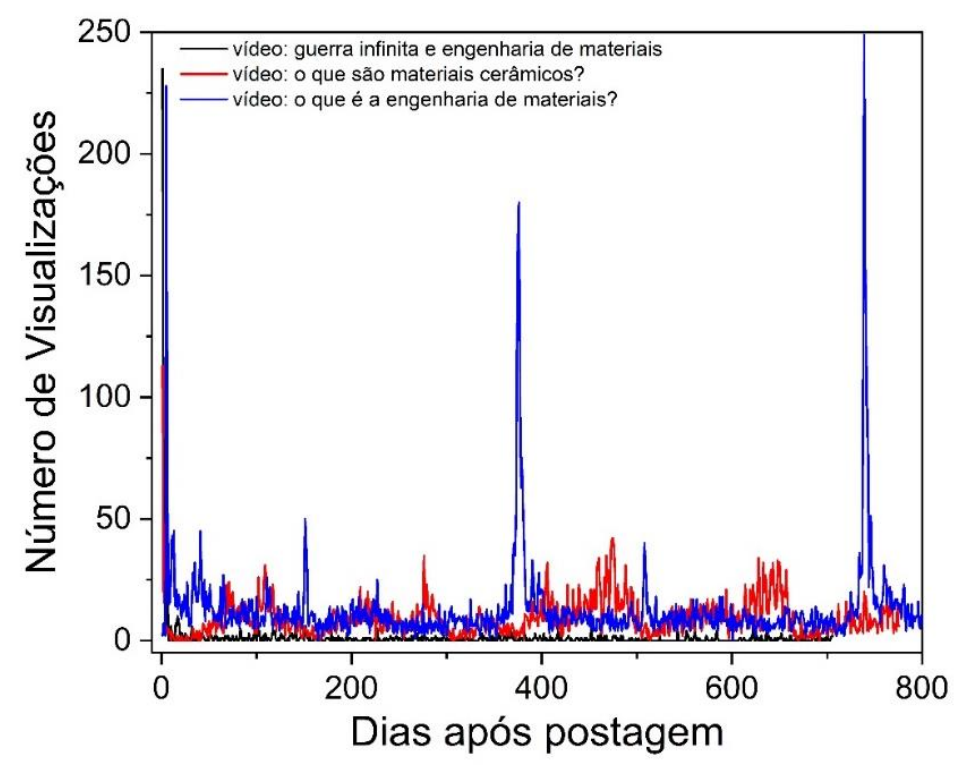

Fonte: Próprio Autor.

Já para o segundo vídeo (Materiais cerâmicos - Engenharia de Materiais), o comportamento da curva não é caracterizado por picos bem definidos como no primeiro vídeo, mas sim picos menos distribuídos por todo o período de veiculação do vídeo. Esse comportamento pode ser observado a partir da curva apresentada na figura 1.

Para esse vídeo, observa-se um interesse durante todo o período de veiculação do vídeo, possivelmente advindo de estudantes recém ingressos no curso de graduação em engenharia de materiais que procuram pelo tema, e de alunos que tem interesse em ingressar no curso e querem saber mais sobre a área. 
O terceiro vídeo, por sua vez, intitulado (Como vencer uma guerra infinita com engenharia), tem o comportamento das visualizações apresentado na figura 1 . Onde pode ser observado um pico de visualizações no momento de lançamento do vídeo, e um decaimento muito grande no restante dos dias de veiculação do vídeo.

Essa avaliação é mais complexa que as anteriores devido ao principal meio de veiculação desse vídeo não ser o YouTube, mas sim o Facebook, onde atingiu 2500 visualizações. Nesse veículo de divulgação não temos acesso a ferramenta analytics, mas a partir de observações dos autores desse trabalho, notou-se que 2000 visualizações foram atingidas no primeiro mês de postagem do vídeo no veículo. Sendo assim é possível concluir que esse tipo de conteúdo, produzido pensando em um assunto que está em alta em um momento específico, adquire visualizações rápido, porém perdem a visibilidade quando o assunto não está mais em alta.

Todos os demais vídeos postados no canal do Youtube apresentaram um número de visualizações de aproximadamente 0,693 visualizações diárias num período de 700 dias. Todos esses vídeos apresentaram um pico nos 10 primeiros dias após sua postagem, sendo que após esse intervalo de tempo o número de visualizações foi reduzido drasticamente.

A Figura 2 nos mostra o meio de acesso pelo qual os usuários acessam aos vídeos de maior número de visualizações do canal "Engenheiros do Amanhã". De acordo com a Figura 2 é possível notar que a maior porcentagem de acessos ocorre através de pesquisas sobre o tema na própria plataforma digital Youtube para os vídeos que explicam do que se trata a engenharia de materiais e os materiais cerâmicos. No caso do vídeo que relaciona a engenharia de materiais com um tema contemporâneo da cultura pop podemos perceber que o maior volume de acessos ocorreu através de links externos ao canal. Esse resultado sugere que o emprego de ferramentas digitais de divulgação cientifica além do Youtube (como por exemplo o Facebook e Instagram) são estratégias que se complementam como uma solução para ampliar o acesso à informação disponibilizada no Youtube. Esse mesmo fenômeno não foi evidenciado nos outros dois vídeos que descrevem o que é a engenharia de materiais e o que são os materiais cerâmicos, pois os mesmos não tiveram a mesma exposição na mídia que o vídeo sobre a engenharia de materiais e a guerra infinita.

Figura 2: Origem de acessos dos usuários que acessam o canal "Engenheiros do Amanhã"

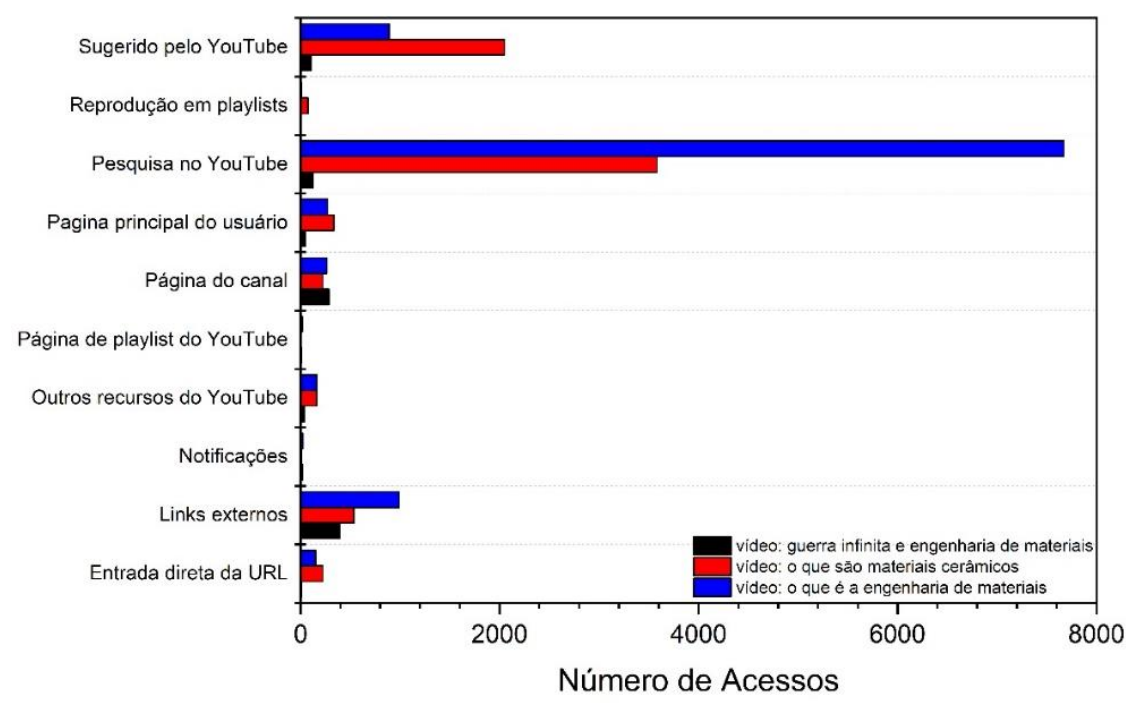

Fonte: Próprio autor. 
Outras informações que podem contribuir para elaboração de estratégias de divulgação científica e popularização de conceitos de engenharia foram identificados como a idade média dos usuários ser compreendida entre 18 e 24 anos (79,9\% das visualizações), o gênero predominante ser o masculino (77,8\% das visualizações) e o tipo de dispositivo empregado durante a visualização do vídeo ser o celular (68,3\% das visualizações). Estes dados também foram extraídos do Youtube Analytics para o vídeo "O que é a engenharia de materiais?”. Até o presente momento, este vídeo foi exibido durante 366,8 horas. Os demais vídeos do canal ainda não possuem essas informações devido a restrições impostas pelo próprio Youtube para vídeos com número de visualizações abaixo de 10 mil.

\section{CONSIDERAÇÕES FINAIS}

O projeto teve um excelente aproveitamento quanto a difusão da engenharia de materiais. Houve um engajamento satisfatório de alunos que procuravam os autores para conversar a respeito da engenharia e de alunos que conversaram entre si sobre o tema, principalmente após a produção do último vídeo sobre a ciência no mundo dos super heróis.

Trazer a ciência para o universo do entretenimento é uma ótima estratégia, pois tira a carga de aprendizado do ouvinte e ao mesmo tempo faz com que ele desperte o interesse em aprender. A partir dos dados coletados, pode-se traçar uma estratégia de criação de conteúdo que produza o máximo de difusão durante todo o ano. Essa estratégia pode ser baseada na produção frequente de conteúdo relacionado a entretenimento, alternada com conteúdo mais teórico de dentro da engenharia, dessa forma, se aproveita os assuntos em alta para obter uma alta difusão de informações em um período, e produz-se conteúdo que é veiculado homogeneamente durante o ano.

A partir da produção desse tipo de conteúdo, espera-se a geração de maior interesse pelo tema, e possivelmente uma menor taxa de evasão dos cursos de engenharia, somada a uma maior procura pelo curso.

Além dos resultados já obtidos, a criação do canal no Youtube pode ter sido um começo pra um projeto maior que tende a crescer e futuramente ter uma importância ainda maior.

\section{Agradecimentos}

Os autores agradecem o apoio da UFLA, FAPEMIG (APQ 04128-15) e CNPQ no desenvolvimento deste trabalho, através da concessão de bolsas de pesquisa e aporte financeiro.

\section{REFERÊNCIAS}

ASLAM, S. YouTube by the Numbers : Stats, Demographics \& Fun Facts. Disponível em: https://www.omnicoreagency.com/youtube-statistics/. Acesso em: 15 abr. 2020.

BAGGI, C. A. dos S.; LOPES, D. A., Evasão E Avaliação Institucional No Ensino Superior: Uma Discussão Bibliográfica. Revista da Avaliação da Educação Superior, Campinas p. 355-374, 2010.

FERNANDES, Danilo Óscar Henriques. Divulgação Científica Utilizando Vídeo e Áudio através da Web. 2009. 57 f. Dissertação (Mestrado) - Faculdade de Engenharia, Universidade do Porto, Porto, 2009.

GAIOSO, N. P. L. O fenômeno da evasão escolar na educação superior no Brasil. 2005. 75 
"Os desafios para formar hoje o engenheiro do amanhã"

f. Dissertação (Mestrado em Educação) - Programa de Pós-Graduação em Educação da Universidade Católica de Brasília, Brasília, 2005.

IAMARINO, A. Nerdologia - YouTube. 2010. Disponível em: https://www.youtube.com/user/nerdologia. Acesso em: 15 abr. 2020.

LIMA, M. de O.; VIANA, G. M. R. Divulgação científica: responsabilidade e importância, Revista Pan-Amazônica de Saúde, v. 8, n. 4, p. 7-8, 2017.

NASCIMENTO, P. M. Canal do Pirulla - YouTube. 2006. Disponível em: https://www.youtube.com/user/Pirulla25. Acesso em: 15 abr. 2020.

PEREIRA, M. C., et al. Evitando evasão em cursos de engenharia: um estudo de caso in XXXIV COBENGE, 2006, Anais. Passo Fundo, 2006.

QUINTANILHA, L. F. Inovação pedagógica universitária mediada pelo Facebook e YouTube: uma experiência de ensino-aprendizagem direcionado à geração-Z. Educar em Revista, n. 65, p. 249-263, 2017.

VELHO, Raphaela Martins Guedes de Azevedo. O Papel Dos Vídeos De Ciência Na Divulgação Científica: O Caso Do Projeto Sciencevlogs Brasil. 2011. Dissertação (Mestrado) Universidade Estadual de Campinas, Instituto de Estudos da Linguagem, Laboratório de Estudos Avançados em Jornalismo. 2011.

SILVA, L. P.; CECÍLIO, S. A mudança no modelo de ensino e de formação na engenharia, Educação em Revista, n. 45, p. 61-80, 2007.

\section{DIGITAL TOOL FOR SCIENTIFIC COMMUNICATION IN MATERIALS ENGINEERING: YOUTUBE CHANNEL ENGENHEIROS

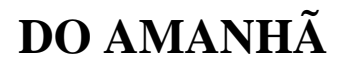

Abstract: The present work aims to present the results (reach and public profile) obtained through the production of scientific dissemination content of the materials engineering course. All content produced was published on the digital platform "youtube". This work shows the reach and profile of the public that accesses the YouTube channel "Engenheiros do Amanhã". Digital tools have proven to be effective for disseminating engineering content to young audiences and students recently enrolled in the materials engineering course in order to reduce dropout rates in engineering courses and increase public awareness of the role of the engineer in Brazilian society. This work was developed around the production and publication of audiovisual content for the YouTube channel "Engenheiros do Amanhã", with all the content as produced by students recently enrolled in the Materials Engineering course at the Federal University of Lavras, in the period between 2017 and 2019. The experience reported is based on the description of the production process of the materials and the work carried out in the laboratories, at home and during some lectures taught by the professor responsible for the discipline. This work presents important data related to visualizations, which can be used to 
"Os desafios para formar hoje o engenheiro do amanhã"

develop and apply strategies for disseminating engineering content in a playful and efficient way.

Keywords: Scientific communication. Digital platforms. Knowledge Diffusion. Materials Engineering 